\title{
GNAO: an MCAO facility for Gemini North
}

Gaetano Sivo $^{\mathrm{a}}$, David Palmer ${ }^{\mathrm{b}}$, Julia Scharwächter ${ }^{\mathrm{b}}$, Morten Andersen ${ }^{\mathrm{a}}$, Natalie Provost ${ }^{\mathrm{a}}$, Eduardo Marin ${ }^{\mathrm{a}}$, Marcos van Dam ${ }^{\mathrm{c}}$, Brian Chinn ${ }^{\mathrm{a}}$, Emmanuel Chirre ${ }^{\mathrm{a}}$, Charles Cavedoni ${ }^{\mathrm{b}}$, Thomas Schneider ${ }^{\mathrm{b}}$, Stacy Kang ${ }^{\mathrm{b}}$, Paul Hirst ${ }^{\mathrm{b}}$, William Rambold ${ }^{\mathrm{b}}$, Angelic Ebbers ${ }^{\mathrm{b}}$, Pedro Gigoux $^{\mathrm{a}}$, Laure Catala ${ }^{\mathrm{b}}$, Thomas Hayward ${ }^{\mathrm{a}}$, John Blakeslee ${ }^{\mathrm{a}}$, Henry Roe ${ }^{\mathrm{a}}$, Jennifer Lotz ${ }^{\mathrm{d}}$, Scot Kleinman $^{\mathrm{b}}$, Suresh Sivanandam ${ }^{\mathrm{e}}$, Anja Krause ${ }^{\mathrm{f}}$, Mark Ammons ${ }^{\mathrm{g}}$, Chadwick Trujillo ${ }^{\mathrm{h}}$, Chris Packham $^{\mathrm{i}}$, Franck Marchis ${ }^{\mathrm{j}}$, Julian Christou ${ }^{\mathrm{k}}$, James Jee ${ }^{\mathrm{l}}$, John Bally ${ }^{\mathrm{m}}$, Mike Pierce ${ }^{\mathrm{n}}$, Thomas Puzia $^{\circ}$, Paolo Turri ${ }^{\mathrm{p}}$, Hwihyun Kim ${ }^{\mathrm{a}}$, Meg Schwamb ${ }^{\mathrm{b}}$, Trent Dupuy ${ }^{\mathrm{b}}$, Ruben Diaz ${ }^{\mathrm{a}}$, Rodrigo Carrasco $^{\mathrm{a}}$, Benoit Neichel ${ }^{\mathrm{q}}$, Carlos Correia $^{\mathrm{r}}$, Eric Steinbring ${ }^{\mathrm{s}}$, François Rigaut ${ }^{\mathrm{t}}$, Jean-Pierre Véran $^{\mathrm{s}}$, Mark Chun $^{\mathrm{u}}$, Masen Lamb ${ }^{\mathrm{e}}$, Scott Chapman $^{\mathrm{v}}$, Simone Espositow ${ }^{\mathrm{w}}$, and Thierry Fusco ${ }^{\mathrm{x}, \mathrm{q}}$

${ }^{a}$ Gemini South Observatory, La Serena, Chile

${ }^{\mathrm{b}}$ Gemini North Observatory, Hilo, United States of America

${ }^{\mathrm{c}}$ Flat Wavefront, Christchurch, New Zealand

${ }^{\mathrm{d}}$ Gemini Observatory, Tucson, United States of America

${ }^{\mathrm{e}}$ University of Toronto, Toronto, Canada

${ }^{\mathrm{f}}$ University of Chicago, Chicago, United States of America

'Lawrence Livermore National Lab, Livermore, United States of America

hNorthern Arizona University, Flagstaff, United States of America

${ }^{i}$ University of Texas San Antonio, San Antonio, United States of America

jSearch of ExtraTerrestrial Intelligence, Mountain View, United States of America

${ }^{k}$ Large Binocular Telescope Observatory, Tucson, United States of America

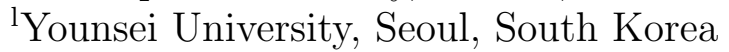

${ }^{\mathrm{m}}$ University of Colorado, Boulder, United States of America

${ }^{\mathrm{n}}$ University of Wyoming, Laramie, United States of America

${ }^{\circ}$ Pontifica Universidad Catolica, Santiago, Chile

pUniversity of California Berkeley, Berkeley, United States of America

${ }^{9}$ Laboratoire d'Astrophysique de Marseille, Marseille, France

${ }^{\mathrm{r}}$ Keck Observatory, Waimea, United States of America

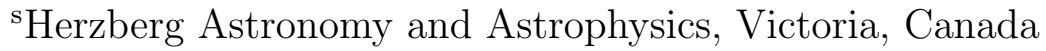

${ }^{\mathrm{t}}$ Australian National University, Canberra, Australia

unstitute for Astronomy, Hilo, United States of America

vDalhousie University, Nova Scotia, Canada

${ }^{\mathrm{w}}$ Osservatorio Astronomico di Arcetri, Florence, Italy

${ }^{x}$ Office national d'études et de recherches aérospatiales, Châtillon, France

\begin{abstract}
Gemini Observatory has been awarded a major funding from the National Science Foundation to build a complete new state of the art multi-conjugate adaptive optics system for Gemini North. The system will be designed to provide an MCAO facility delivering close to diffraction limit correction in the near-infrared over a 2 arcminutes field of view and feed imaging and spectroscopic instruments. We present in this paper the results of the
\end{abstract}

Further author information: (Send correspondence to Gaetano Sivo)

Gaetano Sivo: E-mail: gsivo@gemini.edu, Telephone: 1+56 512205642 
conceptual design phase with details on the new proposed laser guide star facilities and adaptive optics bench. We will present results on the performance simulation assessments as well as the developed selected science cases.

Keywords: Adaptive Optics, Multi-conjugate adaptive optics, GeMS, GNAO, High Angular Resolution, Gemini Observatory

\section{INTRODUCTION}

Gemini Observatory has been awarded a major founding from the National Science Foundation to build a full new state of the art multi-conjugate adaptive optics system for Gemini North. The objective of this award is to enable rapid response and high angular resolution observations in the era of multi-messenger astronomy. Gemini together with the Gemini North Adaptive Optics (GNAO) science team have developed a list of key science cases that will drive the definition of the top level requirements of the AO system in order to answer the observations of these targets. The main requirements for this system are:

- to deliver a close to diffraction limit in the near infrared on a 2 arc minutes field of view;

- obtain a correction of $30 \%$ Stehl ratio in K-band with a goal of $50 \%$;

- obtain a precision astrometry of 0.2 mas across the field (goal of $0.1 \mathrm{mas}$ );

- operate under 1.2" seeing conditions at 0.5 um or better (goal 1.5");

- deliver an output science beam at $\mathrm{f} / 32$;

- sky coverage of $20 \%$ at the galactic pole with 3 natural guide stars (goal of $30 \%$ ) and $60 \%$ with one natural guide star (goal 75\%);

- operable in queue mode with no more than the standard telescope operator + science observer manpower;

- be modular to enable future very wide field ground layer AO mode.

The proposed design to answer these very top level requirements is to build an MCAO system based on the following:

- A laser guide star facility;

- An adaptive optics system;

- A modular real time control system.

The laser guide star system is envisioned to be using 3 Toptica lasers each split in 2 beams to create on-sky a constellation of 6 laser guide stars. Each beam will feed a dedicated side launched laser launch telescope (LLT). 2 constellations are currently considered:

- an hexagon;

- a pentagon with a central star.

We have evaluated the performance obtained with these two configurations and their impact on the science cases. They provide about the same performance on average but differently located across the field. The preferred solution to date is the configuration using the pentagon as the central star can enable high performance science on-axis. One of the main requirements is to build a design that would allow an upgrade of the telescope to obtain a powerful ground layer AO mode. This means we want to build an LGS facility that would allow us to increase the constellation angular size from $1^{\prime}$ (MCAO constellation) to $7^{\prime}$ (GLAO constellation). The Adaptive Optics bench will be equipped with 6 independent high order LGS Shack Hartman wave front sensor of 16x16 
sub-apertures or higher in order to drive two deformable mirrors (DM) at first light and 3 once the Adaptive Secondary Mirror will be integrated to the telescope (outside the scope of this project). Nevertheless, the optical design has to be accounted for this since day one. The plan is to remove the DM conjugated to the ground layer and move it to the mid-altitude layer once the ASM is integrated. The optical design is based on 2 off-axis parabola pairs in order to limit the static distortions into the field induced by the optics. Finally a focal plane NGS WFS is envisioned to control the tip tilt mirror and correct for tip tilt and plate scale mode. Finally a new RTC facility is required in order to have a common platform on this new MCAO facility and the current one in operation at Gemini South on GeMS.

\section{ACKNOWLEDGMENTS}

Based on observations obtained at the Gemini Observatory, which is operated by the Association of Universities for Research in Astronomy, Inc., under a cooperative agreement with the NSF on behalf of the Gemini partnership: the National Science Foundation (United States), National Research Council (Canada), CONICYT (Chile), Ministerio de Ciencia, Tecnología e Innovación Productiva (Argentina), Ministério da Ciência, Tecnologia e Inovação (Brazil), and Korea Astronomy and Space Science Institute (Republic of Korea). Gemini Observatory thanks the National Science Foundation for the GEMMA award. 